\title{
Comparison of Optical Fiber Inertial Vibration Sensors
}

\author{
T. Poczesny*, K. Prokopczuk and A.W. Domanski
}

Faculty of Physics, Warsaw University of Technology, Koszykowa 75, 00-662 Warszawa, Poland

This paper presents the application and comparison of a macrobend seismic optical fiber accelerometer and ferrule-top cantilever fiber sensor (made by Optics11 - Amsterdam, Netherlands) both for long distance vibration monitoring. The sensors utilize typical telecommunication optical transmission systems with specially prepared sensing head. Both optical fiber sensors measure force and acceleration similar to the piezoelectric accelerometers. One of the advantages of these devices is insensitivity to electromagnetic interference because of lack of electrically driven elements in sensing head area. Experiments with wide ranges of frequencies and vibration amplitudes were performed. Main parameters and measurement capabilities of both sensor types were compared. Experimental results show usage possibility of both sensor types in industry and other explosion risk environments for vibration measurement and online monitoring.

PACS: 42.81.Pa, 42.25.Bs, 42.79.Pw

\section{Introduction}

There is a need in chemical and petrochemical industry, gas distribution line system and other harsh environments for fans, motors, turbines, compressors, pump and other equipment vibration measurement and online monitoring [1-3]. Vibration monitoring is mainly based on measurement of displacement or acceleration of tested object. In principle, accelerometer behaves as a damped mass (called seismic mass) on a spring. During acceleration seismic mass is displaced from the neutral position as a result of inertia forces. Measurement of this displacement gives information about acceleration of the object. Modern commercial accelerometers commonly use piezoelectric, piezoresistive, capacitive and micro electro-mechanical systems (MEMS) components to convert mechanical displacement into electric signal. These devices need electrical current in sensing head. This creates potential risk in the area of measurement and makes these devices sensitive to electromagnetic interference.

Presented sensors utilize fiber opto-mechanical transducers for vibration and displacement measurement. They use low-power light sources (e.g. laser diodes, SLD) typically used in telecommunication working on wavelengths of $1310 \mathrm{~nm}$ or $1550 \mathrm{~nm}$. For signal transmission from light source to sensor head and back to photodetector standard single mode (SM) optical fibers are used. Also the sensors heads are all-optical. It provides insensitivity to any electromagnetic interference and allows for elimination of the fire hazard. This design provides generally low-cost construction of multi-point sensing system and online monitoring architecture. All this is an im-

* corresponding author; e-mail: poczesny@if.pw.edu.pl portant advantage which plays significant role in many industrial applications.

Optical fiber macrobend seismic sensor transducer utilizes bend loss phenomenon achieving intensity based signal proportional to acceleration [4]. Continuous optical transmission of light through the head allows for long fiber connections up to $500 \mathrm{~m}$ and longer, depending on the power of light source. The ferrule-top cantilever sensor utilize occurrence of reflection and interference in the sensor head to receive intensity signal proportional to acceleration [5]. In principle both sensors measure signal proportional to inertia force, which acts on structural element of the sensor - similar to the piezoelectric accelerometers - providing useful information about object acceleration, velocity and displacement.

\section{Optical fiber macrobend seismic sensor}

The transducer utilizes the phenomenon of bending losses in optical fibers dependent on radius of the bending [6-8]. It can be described in geometrical approach as a distortion of the angle of incidence due to the geometry deformation (Fig. 1). This causes part of the light to lose its total internal reflection (TIR) propagation mechanism and allows escaping from the optical fiber. In optical fiber mode theory losses are caused by mode shifting due to change of propagation speed (effective refractive index) in order to maintain mode field. Optical fiber macrobend seismic sensor contains bent SM fiber in a loop shape with diameter of $7 \mathrm{~mm}$. To the fiber loop a small $0.3 \mathrm{~g}$ metal sphere is attached. During movement the sphere is deforming the loop through inertia forces [9]. It is acting as a seismic mass. Figure 1 represents schematic illustration of the optical fiber macrobend seismic sensor.

Behavior of the sensor can be described by damped harmonic oscillator equation and its solution is similar to 


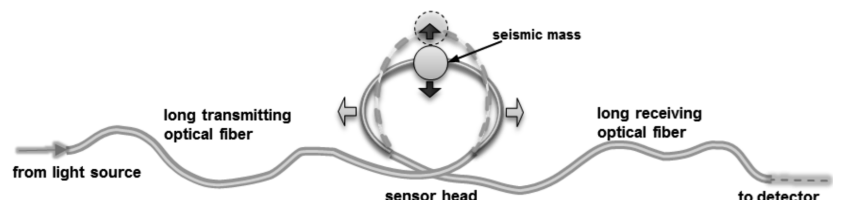

Fig. 1. Optical fiber macrobend seismic sensor schematic and working principle.

$$
x(t)=A \exp (-a t) \sin (\omega t+\varphi) .
$$

The parameter $x(t)$ is proportional to the signal on detector, $A$ is the amplitude of mechanical vibration, while $\omega$ and $a$ are the resonance frequency and dumping coefficient, respectively. The latter two parameters are correlated with the loop diameter and the weight of the seismic mass [10, 11]. Relation of the parameters may be sophisticated due to the impact of the fiber and coating stiffness. In short impulse stimulation damped free oscillations are observed. The resonance frequency for this sensor head configuration is $372 \mathrm{~Hz}$. Figure 2 shows the light spectrum of the SLD light source which is driving the macrobend seismic sensor and light spectrum transmitted by the sensor itself. The attenuation of the fiber macrobend seismic sensing head in presented configuration is in the range of $10-20 \mathrm{~dB}$.

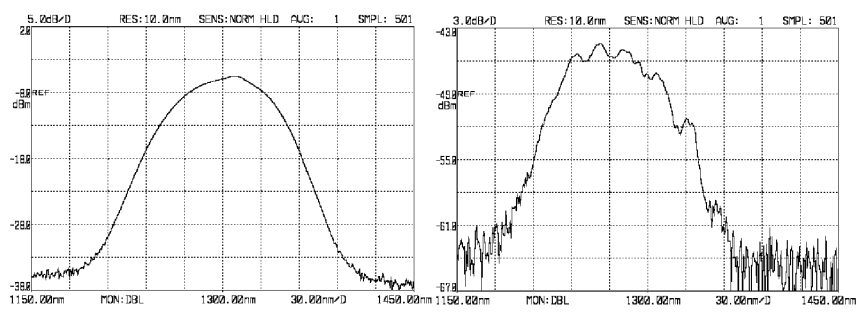

Fig. 2. Spectrum of SLD light source (left) and light spectrum after passing through macrobend sensor (right).

\section{Ferrule-top optical fiber sensor}

The ferrule-top optical sensor is a ferrule made of borosilicate glass in rectangular shape of $3 \mathrm{~mm} \times 3 \mathrm{~mm}$ and $7 \mathrm{~mm}$ height. On top there is carved cantilever made with ps-laser ablation system with precision motion stage. The cantilever itself is $2.8 \mathrm{~mm}$ long, $200 \mu \mathrm{m}$ wide and $30 \mu \mathrm{m}$ thick. The ferrule has a central bore hole to which SM fiber is inserted and glued. This allows illuminating the cantilever from the bottom using optical fiber. The ferrule-top sensor was made and provided by Optics11 [12]. Sensor requires coherent light source allowing interference between light reflected from the face of the fiber and the cantilever (see Fig. 3).

Measured signal is proportional to the distance between the cantilever and optical fiber end. Due to movement the inertia forces tend to bend the ferrule-top cantilever changing the optical patch difference [13]. Simi-

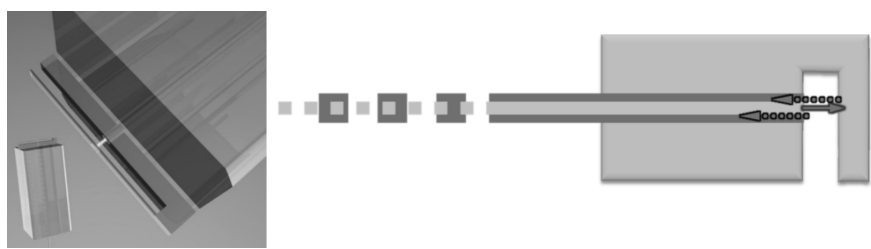

Fig. 3. Schematic of ferrule-top optical fiber sensor.

larly to macrobend seismic sensor the ferrule-top sensor head fulfills Eq. (1). The resonance frequency of $4.2 \mathrm{kHz}$ is an order higher comparing to macrobend seismic sensor due to high stiffness of the glass cantilever.

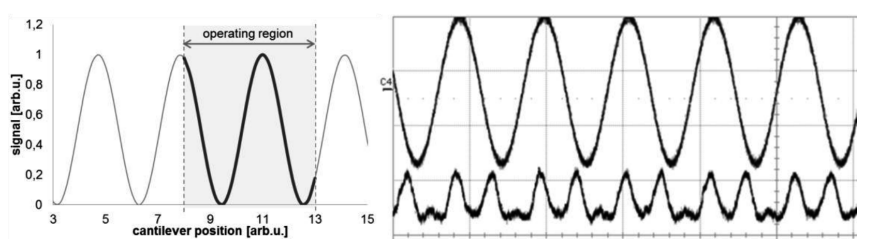

Fig. 4. Example of sensor characteristics (left) and real measurement (right) with single interference fringe passing.

Because of the principle of measurement the response characteristic is periodic interference pattern. It provides high sensitivity within one slope and the number of peaks allows determining the amplitude of cantilever movement. Figure 4 represents an example of real vibration measurement and corresponding theoretical characteristics curve.

\section{Measurement results}

For all experiments we used piezoelectric vibration generator driven by function generator. Signal from the vibration generator is a reference middle curve in all following figures. Telecommunication SM fibers were used for optical connections. The optical fiber macrobend seismic sensor used $1290 \mathrm{~nm}$ SLD light source. The ferrule-top optical fiber sensor used $1550 \mathrm{~nm}$ laser diode as light source connected through 1310/1550 Y-coupler $50 \% / 50 \%$.

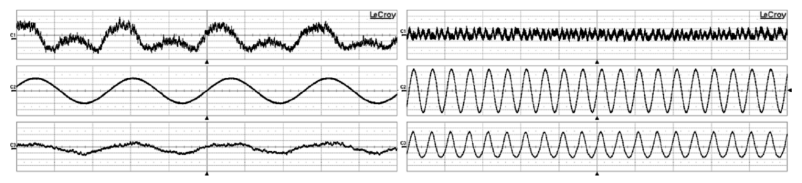

Fig. 5. Low frequency and low amplitude vibration measurement with visible interference fringes passing (middle curve - reference signal; upper curve - macrobend sensor; lower curve - ferrule-top sensor).

Figures 5 and 6 represent vibration measurement accordingly with low frequencies and high frequencies. On 


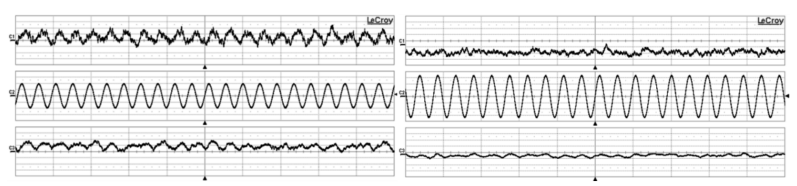

Fig. 6. High frequency vibration measurement: left $11 \mathrm{kHz}$; right $21 \mathrm{kHz}$ (middle curve - reference signal; upper curve - macrobend sensor; lower curve ferrule-top sensor).

top curve in Fig. 5 due to high vibration amplitude the response characteristic of ferrule-top fiber sensor consists of multiply interference fringes (compare Fig. 4) therefore the signal is modulated by nonlinear sensor characteristics.

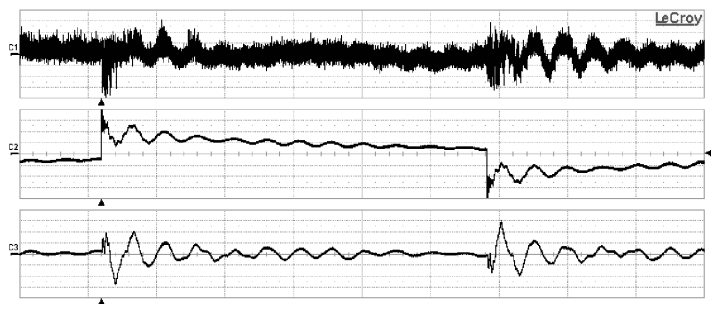

Fig. 7. Impulse vibration measurements (middle curve - reference signal; upper curve - macrobend sensor; lower curve - ferrule-top sensor).

Response for short duration and wide frequency range vibration which represents impulse disturbance is presented in Fig. 7. As measurements show, the signal is correctly measured with negligible resonance effects.

\section{Conclusion}

Experimental results show that both sensors based on standard telecommunication optical fibers with specially prepared sensor head can be used for vibration monitoring in wide range of frequencies. The decay time of resonance vibration is sufficient for impulse vibration measurement. Ferrule-top optical fiber sensor shows higher sensitivity due to utilization of the interference effect and additionally this gives information about cantilever movement. In the case of high vibration amplitude ferrule-top sensor needs more sophisticated data analysis than macrobend sensor due to nonlinear sensor characteristic. Very small size of cantilever makes it vulnerable to any mechanical damage. Optical fiber macrobend seismic sensor gives direct mapping of vibration curve due to its linear response [4]. It does not require the usage of coherent light sources in contrast to the ferrule-top sensor. Both sensors are insensitive to electromagnetic interference and do not create explosion risk. All these features allow them to work in harsh environments.

\section{Acknowledgments}

This work was supported by Polish National Centre for Research and Development (NCBiR) under the grant $\mathrm{N}$ R01 0026 06/2009.

\section{References}

[1] T.R. Wolinski, D. Budaszewski, A.W. Domanski, S. Ertman, G. Goleniewski, M. Wydmanski, Proc. SPIE 6616, 66161U (2007).

[2] Y.R. García, J.M. Corres, J. Goicoechea, J. Sensors 2010, 936487 (2010).

[3] K. Prokopczuk, T. Poczesny, A.W. Domanski, Proc. SPIE 8010, 80100E (2011).

[4] A.W. Domański, T. Poczesny, K. Prokopczuk, P. Makowski, Photonic Lett. Poland 2, 58 (2010).

[5] S. Deladi, D. Iannuzzi, V.J. Gadgil, H. Schreuders, M.C. Elwenspoek, J. Micromech. Microeng. 16, 886 (2006).

[6] D. Marcuse, J. Opt. Soc. Am. B 66, 216 (1976).

[7] R.T. Schermer, J.H. Cole, IEEE J. Quantum Electron. 43, 899 (2007).

[8] P. Wang, Q. Wang, G. Farrell, G. Rajan, T. Freir, J. Cassidy, Microwave Opt. Technol. Lett. 49, 2133 (2007).

[9] T. Poczesny, K. Prokopczuk, A.W. Domanski, Proc. SPIE 8010, 80100D (2011).

[10] T. Poczesny, K. Prokopczuk, P.L. Makowski, A.W. Domanski, Proc. SPIE 8082, 80823L (2011).

[11] P.L. Makowski, R.Z. Morawski, L. Michalik, A.W. Domanski, Measurement: J. Int. Measur. Confeder. 44, 2060 (2011).

[12] http://www.optics11.com/ .

[13] A. Cipullo, G. Gruca, K. Heeck, F. De Filippis, D. Iannuzzi, L. Zeni, Proc. SPIE 7753, 775340 (2011). 